\title{
LES DÉFIS ECONOMIQUES \\ FONDATEURS DE LA NOUVELLE \\ AFRIQUE DU SUD
}

\author{
Pierre Maurice *
}

Le défi economique que confronte à la nouvelle République Sud-Africaine a été recemment reconnu et est révelé par les statistiques officiels. Cette communication discute cette problème: la première partie s'attache à présenter les principaux aspects du défi de la pauvreté; la seconde expose les principaux atouts que possède l'économie sud-africaine pour relever ce défi; et la troisième présente les mesures pour mettre les richesses et atouts existants au service des besoins les plus pressants.

The basic economic challenge that faces a new South Africa has recently been recognised and made evident by official statistics. This article considers that challenge first by looking at poverty in the Republic, second by considering the resources that are available to address poverty, and finally by suggesting measures that can be used to direct the wealth of the nation to meeting the most pressing needs.

\section{INTRODUCTION}

La République Sud-Africaine passait, il y a encore une douzaine d'années, pour un pays riche et industrialisé. Mais les discours et les statistiques officiels occultaient la réalité du sousdéveloppement qui était le lot de la grande majorité de la population de couleur - et donc, la grande majorité de la population tout court - du pays. La puissance économique de l'ancienne Afrique du Sud - celle de la discrimination raciale et du prétendu «développement séparé» (apartheid) - était devenue un mythe - le «mythe fondateur» de l'Afrique du Sud blanche. Avec les bouleversements des années 90 et le changement de régime, la réalité n'est pas «apparue» - elle était connue - mais

* Professeur honoraire des universités, Professeur émérite à l'Université de La Réunion, Coordonnateur de l'Observatoire des Relations Internationales dans l'Hémisphère Sud ORIHS (http://www.upmfgrenoble.fr/orihs).

Le présent texte est celui d'une communication présentée le 29 mars 2003 au colloque sur «Les mythes fondateurs de la nouvelle Afrique du Sud» organisé par le «Groupe de Recherches sur l'Afrique du Sud (GRAS)» de la Faculté des Lettres et Sciences Humaines de l'Université de La Réunion. 
elle a été enfin reconnue et révélée par les discours et, peu à peu, les statistiques officielles, qui s'efforcent désormais de prendre en compte la situation précaire et les retards de développement d'une grande partie de la population. ${ }^{1}$

C'est sur l'immense défi que représente la tâche de réduire ces précarités et retards que cette communication voudrait insister. C'est une tâche tellement cruciale et ardue que l'on peut dire qu'elle constitue le défi fondateur de la nouvelle Afrique du Sud: c'est seulement si elle parvient - à la longue - à combler ces retards et réduire ces inégalités criantes qu'elle méritera (au moins pour l'économiste) le nom de nouvelle Afrique du Sud.

D'où le plan choisi pour cette présentation est le suivant:

I - La première partie s'attache à présenter les principaux aspects de ce défi de la pauvreté relative - à éradiquer, à travers des données concernant la population concernée et ses conditions de vie;

II - La deuxième partie expose les principaux atouts que possède l'économie sud-africaine pour relever ce défi, et qui permettent d'envisager l'avenir avec un certain optimisme;

III - La troisième partie présente les mesures de politique économique et sociale - prises ou à prendre - pour mettre les richesses et atouts existants au service des besoins les plus pressants.

\section{LES DEFIS TENANT A LA PAUVRETE DU PLUS GRAND NOMBRE}

On passera tour à tour en revue:

- Les caractéristiques démographiques et sanitaires de la population - natalité, mortalité, composition par âge, par origine ethnique, migration... - qui déterminent le nombre et la qualité des ressources humaine du pays pour l'avenir;

1 Certaines sources statistiques officielles concernant les pays en développement - tel le Global Development Finance de la Banque Mondiale et la Geographical Distribution of Financial Flows to Developing Countries de l'OCDE - n'incluaient pas, dans le passé, les informations portant sur l'Afrique du Sud, mais les incluent aujourd'hui.

Le PIB par habitant de l'Afrique du Sud était estimé en 2000 à \$9,400 (en PPP ou Parity of Purchasing Power, parité de pouvoir d'achat); par comparaison, celui de la France (continentale) était en 2001 de 23,300 (sans doute 24,000 en 2002) SPA («standard de pouvoir d'achat» qui gomme les fluctuations conjoncturelles de l'Euro), et celui de La Réunion était de 10,900 Euros en 1998 (probablement près de 12,000 en 2002).

Les statistiques sud-africaines actuelles sont de qualité très variable et ont souffert d'une méthodologie politique biaisée, secrète et de faible fiabilité (par exemple les chiffres du commerce extérieur pendant la période des sanctions économiques contre le pays, ceux de la production de diamants, ceux de la population active et du chômage...) qui ont affecté la plupart des domaines de la vie officielle de l'Afrique du Sud dans le passé.

Abréviations utilisées dans ce texte: mn pour million, md pour milliard, R pour rand (la monnaie sudafricaine), PIB pour produit intérieur brut, FMI pour Fonds Monétaire International. 
- Le niveau d'éducation et de formation professionnelle, dont dépendent le taux d'activité et celui du chômage;

- Le manque de logement et la pénurie d'équipements de bien-être et de proximité (crèches, garderies, dispensaires...), qui contribue au développement de la violence et de la criminalité.

\section{A Données Démographiques}

\section{TABLEAU 1 : DONNEES DEMOGRAPHIQUES}

Population (en mn, milieu d'année 2002)

45.5

Taux de croissance de la population (\%) en 2002

Taux de fertilité en $2002 *$

Espérance de vie (années) en 1999

48.0

Taux d'urbanisation (\%) en 2000

* Nombre moyen d'enfants par femme en âge de procréer

Source : Statistics South Africa; World Bank; African Development Indicators

\section{La population totale et ses caractéristiques}

La population d'Afrique du Sud était estimée à 45,5 mn d'habitants en 2002. Le taux moyen de croissance de la population est élevé (par comparaison avec les pays européens): $2 \%$ par an - de même que le taux de fécondité (nombre d'enfants par femme en âge de procréer): $2,9 \%{ }^{2}$ Une décomposition par âge de cette population révèle une population jeune, qui excède les capacités d'accueil du système scolaire et induit rapidement un taux de chômage élevé. ${ }^{3}$

La population continue d'être décomposée selon les trois grands critères traditionnels:

La zone d'habitation, urbaine (52,6\%) ou rurale $(47,4 \%)$;

L'origine ou le mélange ethnique: origine africaine (78\%), blanche (10\%), population «colorée» ou métisse Africains/Blancs (8,6\%), origine indienne ou asiatique (2,5\%), et non spécifiée (moins de 1\%). Les «Africains» sont l'écrasante majorité dans sept des neuf provinces - les métisses sont majoritaires dans celles de Northern Cape et Western Cape, les Asiatiques

2 On sait que le grand espoir à La Réunion depuis un bon nombre d'années était de voir baisser ce taux à près de 2; on est parvenu à 2,26 en 1996, après avoir connu un taux de 3,88 en 1974, mais il est remonté progressivement à 2,5 en 2001; variations similaires (avec des taux plus faibles) en France continentale: 2,11 en 1974, 1,73 en 1996, mais 1,90 en 2001.

3 Le recensement le plus récent a débuté en octobre 2001 et les résultats en sont attendus en 2003. 
(Indiens) demeurent nombreux dans le KwaZulu-Natal. La population blanche, qui jouit d'un haut niveau moyen d'éducation et de qualification, est concentrée dans les provinces de Gauteng (ex-Witwatersrand) et Western Cape;

La langue: la population se répartit en pas moins de 11 langues ou dialectes, dont certains ne sont parlées que par à peine plus de $1 \%$ des habitants. Les langues principales sont l'isiZulu $(22,7 \%)$ l'isiXhosa $(17,7 \%)$ et l'afrikaans $(14,3 \%)$, suivies du sepedi $(9,1 \%)$ et de l'anglais $(8,5 \%)$, qui est toutefois la seconde langue d'une large majorité de la population. (Toute la documentation officielle est de fait publiée en anglais et au moins en une autre «langue officielle»).

\section{Les migrations de population}

Elles constituent un phénomène ancien et important en Afrique du Sud: les mines ont longtemps attiré des travailleurs d'autres pays (notamment du Mozambique); mais une immigration moins officielle a toujours eu lieu vers les fermes proches des frontières du pays. L'ouverture des frontières et le relâchement des contrôles au début des années 90 a accru le phénomène. Les flux officiels sont faibles (en provenance du Royaume Uni, de l'Inde et du Zimbabwe), mais on observe un accroissement très important du nombre d'immigrants illégaux, de faible qualification, en provenance des pays africains voisins, à la recherche d'un emploi. Ce processus, qui s'aggrave avec la détérioration des conditions de vie qui prévalent dans ces pays, induit une tendance à la xénophobie dans la population autochtone. La criminalité croissante (cf. infra) est facilement attribuée aux étrangers nouveaux venus. Le gouvernement est contraint de mener une vigoureuse politique de rapatriement et de restaurer les contrôles aux frontières.

L'existence concomitante d'un flux d'émigration (vers l'Australie, la Nouvelle Zélande, le Royaume Uni et les Etats-Unis) n'est paradoxale qu'en apparence: des Blancs - mais aussi des métisses et des Indiens - quittent le pays, sans doute sous la pression d'un sentiment d'insécurité (civile ou de l'emploi) face à l'avenir, mais seul le groupe des employés blancs a diminué. Cette émigration rend plus aiguë la pénurie de main d'œuvre qualifiée. D'autant plus que la lourdeur bureaucratique des services de l'immigration décourage les travailleurs qualifiés étrangers de venir travailler en Afrique du Sud. ${ }^{4}$

\section{L'emploi et le chômage}

Il est très difficile à évaluer, ${ }^{5}$ car un grand nombres d'activités - rurales aussi bien qu'urbaines sont «informelles» et non déclarées, et c'est bien une des caractéristiques des économies sous-

4 Une loi récente, destinée à freiner l'émigration de main d'œuvre qualifiée, a été adoptée l'an dernier après un âpre débat qui a opposé l'Inkhata Freedom Party (dont le chef, Mangosuthu Buthelezi, était le ministre responsable) et l'African National Congress, le parti dominant.

5 Le chiffre du sous-emploi total n'est pas publié depuis 1998, celui de 1997 provient sans doute d'une estimation à partir des résultats du recensement de 1996. 
développées: «petits boulots» à temps partiel, aide à la ferme, à l'échoppe ou à l'atelier, marchands ambulants, taxis collectifs de banlieue, etc. Le secteur «formel» dont les emplois sont déclarés et officiellement recensés - employait 7,5 mn de personnes en 1997 , soit près de $50 \%$ de la population active, et le secteur informel 1,8 mn (dont 0,8 mn d'employés domestiques), soit plus de $12 \%$. Le reste, soit 38\%, représente le sous-emploi (ou «non-emploi») global, c'est à dire les chômeurs et les personnes qui ne cherchent pas vraiment un emploi ou ont renoncé à en trouver un. Les analystes s'accordent à dire que l'un des problèmes structurels les plus critiques du marché du travail en Afrique du Sud est l'excèdent de travailleurs non-qualifiés ou de basse qualification, et la pénurie de travailleurs hautement qualifiés, qui en font un marché dual.

Il est tout à fait clair que le chômage - total ou partiel, réel ou déguisé, plus résigné que volontaire - de ceux qui cherchent un emploi et des faux «inactifs» résulte avant tout du manque de scolarisation et de formation d'une grande majorité de la population noire, qui est en même temps mal logée, victime désignée des épidémies, mal soignée, et encline à la violence et à la criminalité, produits amers de la pauvreté et du désœuvrement.

TABLEAU 2: RESSOURCES EN MAIN D'EUVRE*

\begin{tabular}{|c|c|c|c|c|c|}
\hline & \multicolumn{5}{|c|}{ (en milliers; estimations en milieu d'année) } \\
\hline & 1994 & 1995 & $1996 * * *$ & 1997 & 1998 \\
\hline TOTAL & 14,187 & 14,501 & 14,911 & 15,149 & 15,484 \\
\hline hommes & 7,573 & 7,754 & 7,990 & 8,127 & 8,321 \\
\hline femmes & 6,614 & 6,747 & 6,921 & 7,022 & 7,163 \\
\hline Secteur officiel & 7,971 & 8,069 & 7,590 & 7,548 & nd \\
\hline $\begin{array}{l}\text { Services sanitaires, sociaux \& socio- } \\
\text { culturels** }\end{array}$ & 2,685 & 2,872 & 1,813 & 1,647 & nd \\
\hline Industries & 1,212 & 1,173 & 1,233 & 1,176 & nd \\
\hline Commerce, restauration et logement & 1,369 & 1,547 & 1,207 & 1,083 & 2,079 \\
\hline Agriculture, forêt et pêche & 1,421 & 965 & 606 & 637 & 1,099 \\
\hline $\begin{array}{l}\text { Finance, immobilier \& services aux } \\
\text { entreprises }\end{array}$ & 388 & 470 & 614 & 503 & 931 \\
\hline Transport, entrepôt et communication & 362 & 395 & 396 & 384 & 539 \\
\hline Construction & 349 & 396 & 385 & 370 & nd \\
\hline Electricité, gaz \& eau & 75 & 77 & 109 & 97 & nd \\
\hline Secteur informel & 1,174 & 1,162 & 979 & 1,840 & 2,705 \\
\hline dont: employés domestiques & 729 & 685 & 688 & 778 & 799 \\
\hline $\begin{array}{l}\text { Total des sans-emploi (définition } \\
\text { large) }\end{array}$ & 5,042 & 5,270 & 6,342 & 5,761 & nd \\
\hline Chômage recensé**** & 271 & 273 & 296 & 310 & 356 \\
\hline $\begin{array}{l}\text { * Population économiquement active } \\
\text { ** Rendus aux ménages (personnes } \\
\text { et collectivités) }\end{array}$ & $\begin{array}{l}\text { ésultats d } \\
\text { Dans les s }\end{array}$ & u recens & & & \\
\hline
\end{tabular}

Sources: Statistics South Africa, Mid-Year Estimates; South African Reserve bank, Quarterly Bulletin. nd : chiffres non disponibles. 
La santé

C'est un domaine qui reflète tout particulièrement l'héritage de la longue période de discrimination raciale (apartheid). Le taux de mortalité infantile est très élevé (au niveau du Bangladesh) dans les familles de souche africaine, mais celui des Blancs est semblable à celui des pays de l'OCDE. Le gouvernement a déjà consenti des efforts considérables en développant (et rendant gratuit) le système de soins et de médecine préventive. Ces dépenses nouvelles ont été controversées, car elles ont paradoxalement nui au financement de l'enseignement dans les hôpitaux qui, historiquement, a produit un excellent standard de qualification et de recherche médicales. Les contraintes budgétaires rendent extrêmement difficile la fourniture de services médicaux de haute qualité. Le problème de la capacité d'accueil en établissements de soins est également très aigu dans les zones rurales, et l'extension du SIDA exerce une pression croissante sur le système de santé. Comme pour de nombreux services en Afrique du Sud, un système parallèle privé existe, mais il est également dans une situation tendue car il soulève un gros problème d'assurance médicale.

Le combat contre le Sida est rapidement devenu l'un des plus grands défis sociaux et économiques de l'Afrique du Sud, et fait sentir son impact sur tous les systèmes de santé, d'éducation, de bien-être et de finances publiques. Cette maladie est en outre devenue une affaire politique majeure, à la fois à l'intérieur et dans les arènes internationales, depuis que le Président Thabo Mbeki a épousé la thèse dissidente selon laquelle le virus HIV et la maladie du Sida ne sont pas liés. Des figures politiques éminentes - telles Nelson Mandela - ont vivement critiqué cette conception du gouvernement. La Cour Constitutionnelle a déclaré que le médicament anti-rétroviral Névirapine devait être disponible pour toute femme enceinte afin d'empêcher la transmission de la maladie de la mère à l'enfant, et pas seulement dans les 18 zones d'expérimentation, comme l'avait décidé le gouvernement. Cette décision est intervenue un an après le début de la Campagne d'Action en faveur du Traitement du Sida menée devant la Haute Cour contre la politique du gouvernement en matière de vaccination anti-rétrovirale.

De nombreux rapports établissent aujourd'hui que le développement du Sida a de grandes chances d'avoir des conséquences catastrophiques non seulement sur la mortalité, mais aussi sur la structure démographique du pays (35\% du groupe d'âge 30-34 ans seraient affectés lorsque l'épidémie aura atteint son sommet en 2006).

\section{B L'Education}

\section{La scolarisation}

L'éducation est probablement le facteur le plus important et le plus décisif pour les perspectives d'avenir en Afrique du Sud (et, plus généralement, pour les pays en développement ou connaissant de grands retards de développement). Sous le régime d'apartheid, des politiques d'éducation basées sur la préférence raciale ont sapé les ressources en capacités et compétences du pays, avec toutes les implications de long terme pour le marché du travail et la compétitivité du pays. L'impact potentiel du Sida est naturellement susceptible d'aggraver la situation. Les données institutionnelles du 
problème sont complexes, et peu de mesures ont été prises avant la nomination du très respecté Kader Asmal au poste de ministre de l'éducation en 1999.

Après une tentative tendue de réviser les cursus scolaires périmés, la version finale du Cursus 2005, dont la publication est imminente (ou a peut-être déjà eu lieu aujourd'hui) va transformer radicalement les méthodes et les contenus de l'enseignement. L'accent est mis en 2003 sur la formation des enseignants, la gestion du système scolaire et l'accroissement des supports pédagogiques fournis aux écoles.

\section{L'enseignement supérieur}

En juin 2002 le ministre a fait connaître ses projets concernant une réorganisation du système d'enseignement supérieur, établis sur la base d'une large consultation commencée dès le début des années 90. Les 21 universités et les 15 instituts de technologie existants dans le secteur tertiaire seront renforcés, en vue de développer des qualifications adaptées aux besoins en ressources humaines du pays. ${ }^{6}$

En dépit de l'importance des réformes engagées ou annoncées, le gouvernement a encore besoin de procéder à une analyse approfondie des moyens d'adapter le système éducatif aux besoins des plus pauvres.

6 En vue de réduire la pénurie de personnels qualifiés sur le marché du travail, $22 \mathrm{mn}$ de rands ont été déboursés pour inciter les étudiants en début d'études à s'engager dans des cursus préparant à des qualifications considérées comme rares par une commission nationale nommée en 2000. La même information a été donnée aux autorités de contrôle de l'immigration, en sorte d'éclairer les choix de recherche d'emploi des immigrants «officiels» et les décisions d'attribution de permis de résidence. 
TABLEAU 3 : NIVEAUX COMPARES DE DEVELOPPEMENT HUMAIN EN 2000

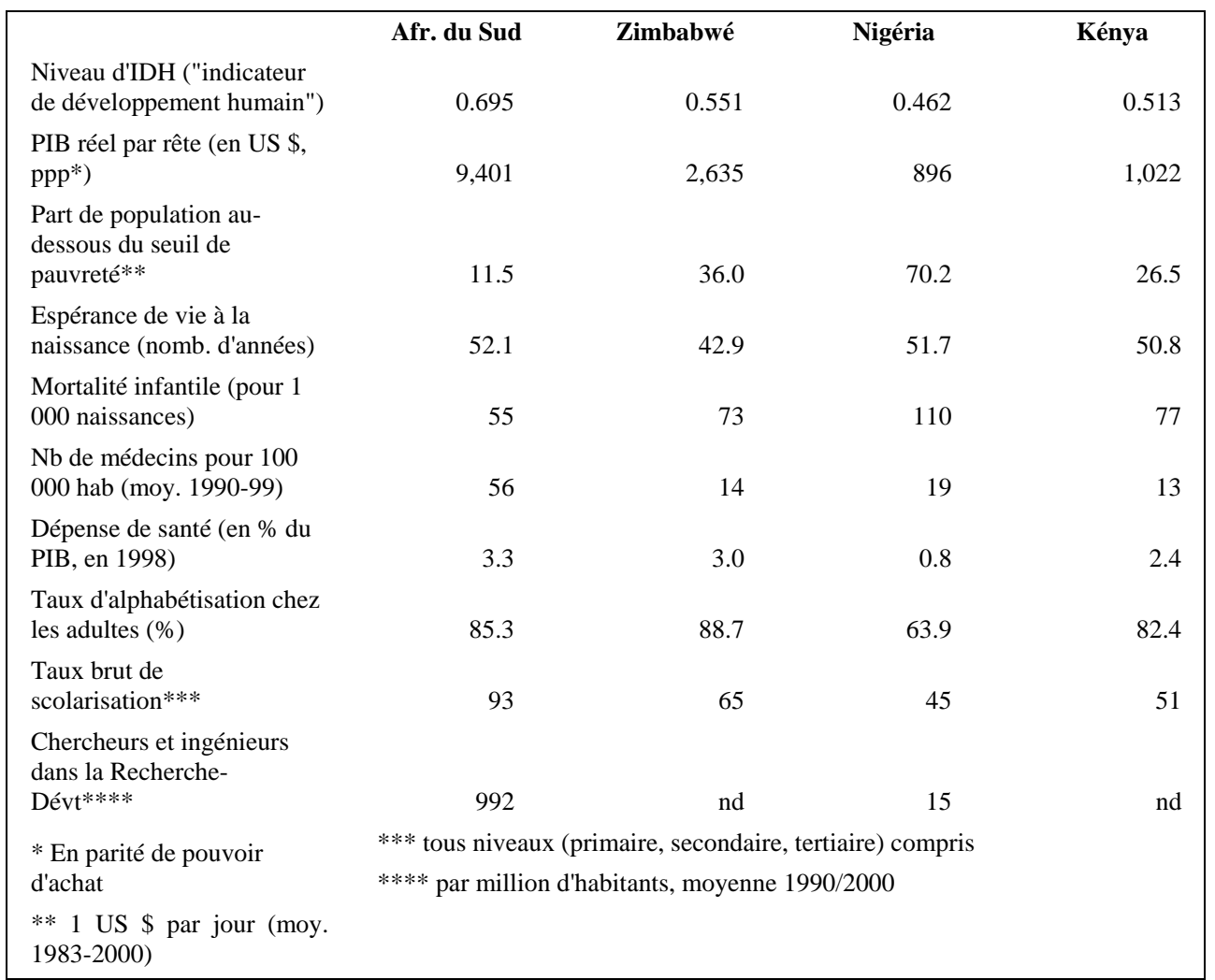

Source: Programme des Nations-Unies pour le Développement (PNUD), Rapport sur le développement humain 2002.

\section{Logement et Conditions de Vie}

1 L'insuffisante quantité et qualité des logements et équipements de proximité

L'habitat des populations d'origine africaine a été longtemps et est encore caractérisé par la précarité et l'insalubrité: les campagnes étaient sous-équipées; les banlieues des villes (townships) qui leur étaient réservées - souvent entourées de barbelés ou de zones abandonnées (no man's land) - étaient constituées de logements, d'équipements et de services municipaux ou de proximité (écoles, dispensaires...) rudimentaires. Depuis la fin de l'apartheid, l'exode rural - qui n'est plus interdit et est difficilement contrôlable - a suscité un afflux de populations en quête d'emplois à la périphérie des grandes villes, entassées dans d'immenses bidonvilles souvent insalubres.

La construction de logements est une des grandes priorités du Programme de Reconstruction et de Développement (cf. III -A). Dès le changement de régime, le gouvernement a signé un accord 
avec les banques et les sociétés de construction, portant sur 2 mds de rands, selon lequel le secteur privé devait en priorité prêter de l'argent aux demandeurs de logement les plus pauvres, le gouvernement s'engageant à indemniser ces institutions au cas où l'insécurité politique les empêcherait de récupérer leurs fonds. Fin 1996 le gouvernement a changé radicalement sa politique en faveur du logement pour accélérer les réalisations, en mettant l'accent sur un partenariat entre les secteurs privé et public et en accordant un plus grand rôle aux gouvernements locaux. Pourtant, les progrès ont été lents, les banques étant toujours réticentes à soutenir le système de financement en faveur des familles les plus pauvres avec des prêts commerciaux. Peu de progrès également dans la tentative de persuader les habitants des «banlieues de la ségrégation» de cesser leur refus de payer les loyers et services sociaux de leurs quartiers, qui grève les finances des gouvernements locaux. En outre, le secteur de la construction a connu une forte réduction, ses capacités s'étant dramatiquement affaiblies, du fait d'équipements périmés et d'une pénurie de main d'œuvre qualifiée, qui ont entraîné la fermeture de nombreuses entreprises. Le redressement de la situation est handicapé par la cartellisation du secteur, qui rend très difficile aux nouveaux arrivants particulièrement les entreprises «noires» de s'introduire sur le marché, envahi de comparses véreux.

Les dépenses du gouvernement en matière d'infrastructure se sont nettement accrues dans les deux derniers budgets, mais la tendance générale au ralentissement de l'activité n'est pas propice à une croissance soutenue dans ce secteur.

\section{La violence et la criminalité}

Les tensions économiques et sociales résultant de l'énorme écart entre les niveaux de vie moyens d'une minorité naguère officiellement privilégiée d'une part, et d'une grande majorité de la population officiellement et systématiquement mise (pendant des décennies) à l'écart du progrès d'autre part, sont certainement responsables, aujourd'hui et sans doute pour longtemps encore, du niveau de violence et de criminalité qu'on observe dans le pays, particulièrement dans la région urbanisée du Gauteng et dans celle de Western Cape. ${ }^{7}$

Dans la première de ces régions, l'insécurité a même affecté l'activité économique et le quartier des affaires, qui ont tendance à se déplacer hors du district central de Johannesburg, vers des quartiers résidentiels plus calmes comme Sandton. Des entreprises ont créé une organisation appelée «Le monde des affaires contre le crime» (Business Against Crime), organisme de surveillance et d'intervention qui combat le crime organisé au niveau local. Le haut niveau de criminalité, et le climat d'insécurité et de crainte qu'elle induit, sont perçus comme l'un des obstacles à la croissance économique, encore que des études faites auprès des investisseurs étrangers donnent une image nuancée et assez diversifiée de ce climat et de cette perception.

7 Dans la région de Western Cape, le crime sévit surtout dans la région de Cape Flats, et est le fait de gangs armés organisés. 
Une partie de cette criminalité a des origines politiques, et reste liée à l'héritage historique de la période de discrimination raciale, à la société violente que ce système a engendrée, et au haut niveau de détention d'armes chez des particuliers. Elle est aussi encouragée en fonction du degré d'impunité dont semblent parfois jouir les auteurs de crimes. ${ }^{8}$

Par dessus tout, l'origine de cette criminalité semble bien se trouver dans les énormes inégalités qui subsistent encore dans ce pays: inégalité à la naissance naguère, inégalité dans les chances d'accéder à l'éducation et au savoir, inégalité dans l'accès au marché du travail, aux revenus élevés et à tout ce qu'ils permettent d'acquérir: santé, logement, confort et bien-être, consideration. ${ }^{9}$ C'est certainement cet ensemble de frustrations qui est la cause profonde - et l'ancienne discrimination raciale l'origine première - des tensions sociales et de la propension à la violence civile, qui créent et entretiennent elles-mêmes un climat d'insécurité.

En définitive, et pour conclure cette partie, les problèmes liés à la situation démographique, sanitaire, éducative, sociale d'une grande majorité de la population ne constituent que les différentes facettes de l'énorme exclusion qu'elles a vécue dans le passé par rapport à la tranche des blancs riches et instruits, et c'est bien la réduction de cette formidable inégalité et de la frustration qu'elle engendre qui est l'immense défi que les dirigeants actuels doivent relever.

Il nous faut voir alors quels sont les richesses et les atouts dont dispose ce pays pour tenter de et, on l'espère, pour réussir à - relever ce défi.

\section{LES ATOUTS TENANT AUX RICHESSES NATURELLES ET ACCUMULEES}

Les atouts dont dispose l'Afrique du Sud actuelle pour relever les défis évoqués plus haut sont de trois ordres:

- Des richesses naturelles et transformées: climat, ressources du sol et du sous-sol;

- $\quad$ Des ressources humaines, tout particulièrement le savoir-faire des élites;

8 La violence politique proprement dite - règlements de comptes entre partis opposés a, quant à celle, fortement décru. L'essentiel de cette violence s'est manifestée au cours des années récentes dans le KwaZulu-Natal et le Gauteng, où le conflit entre l'African National Congress (ANC) et l'Inkhata Freedom Party a coûté la vie à un nombre de personnes estimé entre 14 et 20000 depuis 1994.

9 L'Afrique du Sud est certainement l'un des pays où la répartition des revenus est la plus inégale dans le monde: on peut y observer d'un côté l'abondance des biens dans les grands centres commerciaux et la richesse voire l'opulence des beaux immeubles et des résidences de campagne, de l'autre un habitat et des conditions de vie qui traduisent le niveau de pauvreté d'un pays sous-développé. En 1996, 57\% de la population vivait au niveau du seuil de pauvreté (ou au-dessous), et les statistiques officielles indiquaient que $36,2 \%$ de la population en âge de travailler étaient soit au chômage soit «inactifs» c'est à dire ne cherchaient plus activement un emploi ou avaient renoncé à en chercher un. 
- Des équipements publics et privés d'infrastructure: équipements urbains, parc immobilier, moyens de transport et de communication modernes.

\section{A Les Richesses Naturelles et Transformées}

\section{Le climat}

Dont jouit l'Afrique du Sud explique d'évidence l'installation précoce et abondante d'Européens dans ce pays. Les côtes occidentales et méridionales ont un climat tempéré, tandis que la côte orientale est subtropicale. Il y a de larges bandes de terres arides à l'intérieur, le Karoo dans les provinces du Cap, une partie du plateau africain qui s'étend jusqu'au bassin de Kalahari avant de s'élever dans le Highveld jusqu'à une altitude de 1500 à 2000 mètres. Un autre trait de la topographie est l'existence du Grand Escarpement qui entoure le flanc Est du plateau, culminant vers les sommets élevés de la chaîne du Drakensberg.

La diversité des conditions climatiques et des sols a permis d'adapter une large variété de cultures: les grandes fermes livrées au maïs et au blé dominent dans le Highveld, mais ailleurs les productions de légumes et de fruits tempérés et tropicaux, ainsi que la canne à sucre (dans le KwaZulu-Natal) augmentent régulièrement. Le vin, de grande qualité, prédomine dans le Western Cape. Environ 13\% des terres sont arables, tandis que 67\% sont couvertes de pâturages (consacrés aux bovins, ovins, caprins et autruches). Ces productions, qui ne pèsent que $5 \%$ du PIB, continuent de fournir de nombreux emplois en zones rurales, particulièrement dans les anciennes «patries ethniques» (homelands) et, à l'état brut ou par l'intermédiaire d'industries agro-alimentaires, sont sources régulières de recettes d'exportation (30\% du commerce extérieur de produits, or exclu).

La forêt (conifères) est réduite (6\% des terres) mais s'étend; la pêche est de faible importance aujourd'hui, en partie en raison de la surexploitation passée des ressources côtières (anchois et colin du Cap) qui ont conduit à l'imposition de quotas très stricts dans les années 80.

\section{Les sources d'énergie}

(Charbon, électricité hydraulique, pétrole produit à partir du charbon par SASOL) sont abondantes et bien exploitées. ESKOM, le producteur nationalisé d'électricité, est aussi le fournisseur d'énergie pour une bonne partie du continent africain, l'un des mieux équipés et des moins chers du monde (ce dernier avantage est peut-être moins dû à l'efficience managériale de la société qu'aux avantages fiscaux concédés par le gouvernement). La consommation d'électricité par habitant (pour ceux qui sont desservis) est comparable à celle du Royaume-Uni.

L'Afrique du Sud possède près du Cap la seule centrale nucléaire existant en Afrique, qui alimente en courant électrique toute la province de Western Cape. Sa durée de vie pourrait encore 
être de 30 à 40 ans, mais il n'existe actuellement aucun projet d'expansion de l'énergie nucléaire ${ }^{10}$ (le programme nucléaire militaire a été stoppé après 1994).

3 Les activités minières

L'Afrique du Sud possède d'énormes ressources minières: $80 \%$ des réserves mondiales de manganèse, $56 \%$ pour les minerais du groupe platine, $35 \%$ pour l'or et $68 \%$ pour le chrome.

TABLEAU 4 : RANG DE L'AFRIQUE DU SUD DANS LES RESERVES ET LA PRODUCTION MONDIALES DE MINERAIS 2000

\begin{tabular}{|lcc|}
\hline & Réserves & Production \\
Or & 1 & 1 \\
Métaux du groupe platine & 1 & 1 \\
Minerai de chrome & 1 & 1 \\
Vanadium & 1 & 1 \\
Alumino-silicates & 1 & 1 \\
Minerai de manganèse & 1 & 1 \\
Vermiculite & 2 & 1 \\
Uranium & 4 & 9 \\
Diamants & 5 & 5 \\
Charbon & 5 & 9 \\
Nickel & 6 & 8 \\
Minerai de fer & 6 & 9 \\
Aluminium & nd & 9 \\
\hline
\end{tabular}

Source : Département des Mines et de l'Energie, Bureau des Minéraux.

L'or comptait pour 13\% du total des exportations à la mi-2002 (+ 11\% par rapport à $\left.2001^{11}\right)$ contre $50 \%$ en 1980 . Les mines sud-africaines sont les champions mondiaux de la technologie minière en grande profondeur. Mais la production des mines très profondes devient de plus en plus anti-économique (les coûts s'accroissent et la teneur du minerai diminue), ce qui a conduit à la fermeture de groupes miniers dominants, ou la vente des opérations les moins rentables, ou encore la fusion avec une activité de traitement industriel.

Le groupe des métaux de platine a surpassé l'or en 2002 et constitué la plus grosse exportation en valeur du pays. Du coup les activités minières d'Afrique du Sud en général se sont lancées dans

10 Certaines mines d'or continuent de produire de l'uranium comme sous-produit, destiné à l'exportation, mais les chiffres son tenus secrets.

11 La hausse du prix de l'or et la dépréciation du rand expliquent qu'une baisse de la production en 2002 se soit accompagnée d'une augmentation des recettes d'exportation. L'AngloGold d'Afrique du Sud est le plus gros producteur mondial. 
des programmes d'expansion. ${ }^{12}$ D'autre part, la hausse des prix des métaux précieux entre la fin des années 90 et les premières années du $21^{\mathrm{e}}$ siècle a attiré dans cette branche bon nombre de nouveaux candidats producteurs, et cette tendance pourrait se poursuivre à moyen et même long terme.

En matière de diamants, De Beers est le plus gros producteur mondial, et contrôle l'offre et la distribution mondiale à travers sa filiale Diamond Trading Company basée à Londres.

Un grand nombre d'activités manufacturières sont engagées assez récemment dans le traitement et la transformation des minerais, fer et manganèse inclus. Le raffinage et la transformation des minerais en métaux (or, aluminium et autres) a attiré de larges investissements et est devenue une source croissante de recettes d'exportation.

\section{Les activités industrielles et le tourisme}

Les activités manufacturières comptent pour environ 1/5 du PIB. Ce secteur qui, au départ, s'était développé à l'abri d'un régime protectionniste, a dû opérer d'importantes restructurations pour devenir compétitif sur le marché mondial depuis que la libéralisation du commerce extérieur est mise en œuvre. Ce processus a entraîné la contraction d'activités non compétitives telles que le textile et la confection, et la croissance d'industries d'exportation, notamment les véhicules à moteur. Le secteur industriel dans son ensemble a connu une régression entre 1988 et 1994, mais a enregistré une nouvelle expansion depuis lors, y compris de sa part dans les exportations totales, malgré la faible croissance générale de l'activité et la pénurie de main d'œuvre qualifiée, - mais grâce, sans doute, à un taux de change réel compétitif induit par la baisse de la valeur externe du rand en 2001. Malheureusement, même si elle reste le plus gros employeur, c'est dans l'industrie que se sont produites les principales pertes d'emploi de ces dernières années, du fait de ses restructurations. Cette industrie est aujourd'hui très intensive en capital, et détient des capacités de production à même de répondre à des accroissements de la demande. Elle repose sur les activités minières, la transformation des minerais, la production d'énergie, la chimie lourde, le fer et l'acier, ${ }^{13}$ l'aluminium. En revanche, elle est faible dans les domaines des biens d'équipement et des biens intermédiaires.

L'Afrique du Sud a accru sa part de marché dans le tourisme mondial depuis le début des années 90, bien qu'il ne contribue encore, en 2002, que pour 3\% du PIB (alors que la moyenne dans le monde tourne autour de 6\%). En 2000 le gouvernement a lancé une opération de promotion du tourisme en Afrique du Sud, concentrée sur les 6 plus grands marchés: 5 pays européens (France, Allemagne, Italie, Pays-Bas et Royaume-Uni) et les Etats-Unis, campagne qui a donné depuis lors des résultats très satisfaisants.

12 Anglo Platinum a entrepris le plus vaste programme d'expansion, qui accroîtra sa production de platine de 75\% d'ici 2006.

13 L'industrie sud-africaine de l'acier est l'un des 25 premiers producteurs du monde et détient un grand nombre de producteurs extrêmement compétitifs, tel Columbus Stainless. 

TABLEAU 5: PRODUIT INTERIEUR BRUT PAR BRANCHE \& VARIATIONS ANNUELLES

\begin{tabular}{|c|c|c|c|c|c|c|c|c|c|c|}
\hline \multicolumn{11}{|c|}{ (en millions de rand, à prix constants de 1995; variations en \%) } \\
\hline & 1997 & $\begin{array}{c}\text { Part en } \\
\%\end{array}$ & 1998 & $\begin{array}{c}\text { Part en } \\
\%\end{array}$ & 1999 & $\begin{array}{c}\text { Part en } \\
\%\end{array}$ & 2000 & $\begin{array}{l}\text { Part } \\
\text { en } \%\end{array}$ & 2001 & $\begin{array}{l}\text { Part } \\
\text { en } \%\end{array}$ \\
\hline $\begin{array}{l}\text { Agriculture, forêt et } \\
\text { pêche }\end{array}$ & 24,153 & 4.51 & 22,519 & 4.17 & 23,658 & 4.29 & 25,389 & 4.45 & 24,590 & 4.22 \\
\hline Mines et carrières & 35,120 & 6.56 & 34,840 & 6.46 & 34,472 & 6.25 & 33,821 & 5.93 & 33,865 & 5.81 \\
\hline Industries & 110,562 & 20.65 & 108,418 & 20.09 & 108,084 & 19.58 & 113,587 & 19.90 & 116,913 & 20.04 \\
\hline $\begin{array}{c}\text { Electricité, gaz et } \\
\text { eau }\end{array}$ & 20,034 & 3.74 & 20,358 & 3.77 & 20,728 & 3.76 & 20,244 & 3.55 & 20,103 & 3.45 \\
\hline Construction & 16,646 & 3.11 & 17,084 & 3.17 & 16,670 & 3.02 & 17,122 & 3.00 & 17,805 & 3.05 \\
\hline $\begin{array}{c}\text { Commerce (gros \& } \\
\text { détail), restauration, } \\
\text { logement }\end{array}$ & 74,748 & 13.96 & 73,905 & 13.69 & 74,162 & 13.44 & 77,372 & 13.56 & 80,029 & 13.72 \\
\hline $\begin{array}{l}\text { Transport, entrepôt } \\
\text { et communication }\end{array}$ & 50,879 & 9.50 & 54,273 & 10.06 & 58,141 & 10.53 & 61,000 & 10.69 & 63,354 & 10.86 \\
\hline $\begin{array}{l}\text { Finances, assurance, } \\
\text { immob. \& serv. aux } \\
\text { entreprises }\end{array}$ & 91,866 & 17.16 & 96,737 & 17.92 & 104,191 & 18.88 & 110,318 & 19.33 & 114,488 & 19.63 \\
\hline $\begin{array}{l}\text { Services sanitaires, } \\
\text { sociaux \& socio- } \\
\text { culturels }\end{array}$ & 111,312 & 20.79 & 111,564 & 20.67 & 111,826 & 20.26 & 111,900 & 19.61 & 112,203 & 19.23 \\
\hline $\begin{array}{l}\text { Coût des services } \\
\text { financiers imputés }\end{array}$ & 13,971 & & 14,108 & & 14,439 & & 14,925 & & 15,404 & \\
\hline $\begin{array}{c}\text { Services } \\
\text { gouvernementaux }\end{array}$ & 83,043 & & 82,830 & & 82,469 & & 81,758 & & 81,136 & \\
\hline Autres producteurs & 14,298 & & 14,626 & & 14,918 & & 15,217 & & 15,663 & \\
\hline $\begin{array}{l}\text { PIB au coût des } \\
\text { facteurs }\end{array}$ & 535,320 & 100 & 539,698 & 100 & 551,931 & 100 & 570,753 & 100 & 583,350 & 100 \\
\hline $\begin{array}{l}\text { Taux de variation } \\
\text { annuelle réelle }\end{array}$ & nd & & 1.01 & & 1.02 & & 1.03 & & 1.02 & \\
\hline $\begin{array}{l}\text { PIB aux prix du } \\
\text { marché }\end{array}$ & 586,837 & & 591,310 & & 603,841 & & 624,127 & & 638,010 & \\
\hline $\begin{array}{l}\text { Taux de variation } \\
\text { annuelle réelle }\end{array}$ & 2.6 & & 0.8 & & 2.1 & & 3.4 & & 2.2 & \\
\hline $\begin{array}{l}\text { PIB aux prix du } \\
\text { marché par tête (en } \\
\text { rand) }\end{array}$ & 14,291 & & 14,100 & & 14,099 & & 14,287 & & 14,321 & \\
\hline $\begin{array}{l}\text { Taux de variation } \\
\text { annuelle réelle }\end{array}$ & 0.4 & & 1.3 & & 0.0 & & 1.3 & & 0.2 & \\
\hline
\end{tabular}

\section{B Les Ressources Humaines}

L'une des grandes richesses de l'Afrique du Sud est constituée par ses ressources humaines, actuelles et potentielles, et le savoir faire de ses élites. L'enseignement et la recherche universitaires y contribuent de plus en plus; le développement et la bonne gestion des activités de services privés (commerce, services financiers) et publics, et le niveau de compétence de certains responsables et corps intermédiaires (hauts fonctionnaires, syndicalistes...) l'attestent. 



\section{Les universités}

Le système universitaire d'Afrique du Sud est le meilleur de toute l'Afrique, mêmes si la pléthore d'universités et d'instituts techniques (36 en tout) doit être rationalisée et leur nombre ramené à 21, selon les propositions de restructuration du gouvernement. Depuis 1994, les anciennes institutions blanches, telles que l'Université du Witwatersrand à Johannesburg et l'Université du Cap ont inscrit chez elles de plus en plus d'étudiants noirs ou de couleur. Les plus grandes et les plus riches des universités se battent pour se transformer, et les anciennes institutions non-blanches essaient de résister avec de maigres ressources et des effectifs étudiants en baisse.

Au recensement de 1996, 6\% de la population (des Blancs pour la plupart) avaient reçu une formation supérieure. Les étudiants et le personnel enseignant non blancs se sont fortement accrus dans les plus grandes universités au cours de ces dernières années. Pourtant il subsiste un écart très grand entre les attentes des universités et la scolarité rudimentaire de beaucoup d'Africains, ce qui fait que le nombre global de ces derniers qui ont pu être admis dans les universités a, de fait, baissé depuis 1994.

\section{Les services}

L'ensemble des services, privés et publics, proposés aux entreprises et aux particuliers, ${ }^{14}$ pesait 63,4\% du PIB en 2001. Ce pourrait être le signe d'un pays sous-développé - où les activités de production et de transformation de biens seraient rudimentaires, et le secteur informel pléthorique si les secteurs primaire et secondaire marchands n'étaient pas développés et modernes comme ils le sont en Afrique du Sud. Ce chiffre est donc plutôt le signe d'un pays développé, au secteur tertiaire déjà très avancé. Ce qui constitue un atout supplémentaire pour l'avenir du pays.

Les services privés modernes sont déjà très développés et de bonne qualité: il faut citer en particulier le commerce d'import-export, les services liés au tourisme, et les services financiers. Les services publics méritent souvent (mais pas tous) les mêmes qualificatifs, ou celui - plus moderne de «bonne gouvernance»

Le commerce d'import-export, traditionnellement très bien organisé, a longuement souffert des sanctions économiques imposées par la communauté internationale, surtout le commerce extracontinental - le commerce avec les pays voisins n'a jamais cessé, mais il souffre toujours du niveau faible de la demande solvable émanant de ces pays. Les sanctions (et un arrêt de l'endettement), dans les années 80, avaient contraint l'Afrique du Sud à devenir un exportateur net de capitaux (elle enregistrait peu ou pas d'apports nouveaux extérieurs, et ses créanciers exigeaient d'être remboursés), et donc à dégager un surplus important de sa balance commerciale, lequel - compte tenu de la mévente des produits sud-africains imputable aux sanctions - ne pouvait être obtenu que par une réduction drastique des importations et des programmes massifs d'import-substitution. Les

14 «Business services» et «Community, social \& personal services». 
bouleverse-ments politiques intervenus dans les années 90 ont entraîné la fin des sanctions et une large ouverture vers le monde extérieur, étayée par une politique courageuse de libéralisation de commerce, qui a constitué la base d'une intégration (ou ré-intégration) élargie de l'Afrique du Sud dans le commerce et les marchés financiers mondiaux. Ce pays est aujourd'hui un membre actif de l'Organisation Mondiale du Commerce, et de nombreuses organisations économiques régionales et internationals. ${ }^{15}$

Les services financiers sont très développés et de très bonne qualité. Ils contribuent pour $20 \%$ au PIB, et sont les plus avancés de l'Afrique sub-saharienne (les marchés financiers sud-africains sont plus importants, en chiffres d'affaires, que tous les autres échanges sub-sahariens réunis). ${ }^{16}$

Dans un environnement de faible épargne, les ressources de ce secteur ne peuvent provenir que du secteur moderne (mines, énergie et industries) et d'investissements ou placements étrangers. Quatre grandes banques dominent le secteur bancaire (elles représentaient 69,5\% de ce secteur en 2001): ABSA, Standard Bank Investment Corporation (Stanbic), Nedcor et FirstBank (l'ancienne First National Bank), qui ont toutes des liens étroits avec les grandes compagnies d'assurance sur la vie (Sanlam, Old Mutual, Liberty...). Presque toutes les grandes institutions financières du pays gèrent des investissements extra-territorialisés (off shore), et subissent maintenant la concurrence de grandes banques internationales telles que la Citybank ou la JP Morgan Chase, qui sont installées en Afrique du Sud où elles ont parfois racheté des établissements locaux.

Toutes ces institutions interviennent sur le marché financier de Johannesburg Stock Exchange, qui a pris le nom de JSE Securities Exchange en 2001. En janvier 2002, cet organisme a entrepris toute une série de réformes et de remaniements pour améliorer sa compétitivité. En mai, il a signé un accord-cadre avec la Bourse de Londres (London Stock Exchange) accordant aux courtiers londoniens un plus large accès aux titres du JSE grâce à un partage de services technologiques de pointe.

La banque centrale (South African Reserve Bank ou SARB) est indépendante du gouvernement et jouit d'un haut degré d'autonomie dans la détermination de la politique monétaire du pays, dont l'objectif essentiel est de contenir le taux annuel d'inflation dans les marges fixées (entre 3 et 6\% pour les quatre années 2002 à 2005) et de maintenir la stabilité des prix. Son gouverneur actuel (nommé en 1999), M. Tito MBOWENI, ancien ministre du travail, est le premier gouverneur noir de

15 Les premières sont: L'Union Africaine (ex-Organisation de l'Unité Africaine), la SACU (Southern African Customs Union), la SADC (Southern African Development Community), et la Convention de Cotonou (qui fait suite aux accords de Lomé entre l'Union Européenne et les pays ACP). Les secondes sont, outre l'Organisation Mondiale du Commerce, le FMI, la Banque Mondiale, et toutes les organisations du groupe des Nations-Unies (FAO, CNUCED, etc.)

16 Le rapport sur la stabilité du secteur financier produit par le FMI décrivait les marchés financiers sudafricains, dans sa récente mise à jour, comme «hautement développés et équilibrés», et sa structure légale et réglementaire comme «impressionnante par l'étendue de sa couverture». 
la Banque centrale. Les craintes que ce fidèle de l'ANC soit sensible aux pressions politiques se sont révélé infondées.

\section{Le sens de l'intérêt général et du service public des corps intermédiaires et des élites}

L'Afrique du Sud d'aujourd'hui se fait remarquer par un certain sens de la «bonne gouvernance» de ses hauts responsables, ministres hauts fonctionnaires et syndicalistes.

Une certaine crainte s'est manifestée parfois quant au risque de «copinage» politique dans les nominations aux principaux postes de la fonction publique et les contrats signés par le gouvernement. Mais cette tendance n'est en rien comparable avec l'étendue du problème dans de nombreux pays en développement. D'autre part, si une certaine corruption existe (comme le laisse supposer une récente tractation en matière d'armes), ce n'est pas du tout à l'échelle de beaucoup d'autres pays africains. Selon Transparence Internationale, qui calcule un indice de perception de la corruption, l'Afrique du Sud est classée 36 e (à égalité avec la Tunisie) en 2002, dans le classement de 102 pays, avec un score de 4,8 (0 indique une totale corruption, 10 une corruption nulle).

Au gouvernement, $\mathrm{M}$ Trevor Manuel est l'actuel ministre des finances - poste qui fut un temps convoité par M Tito Mboweni. Il s'est très vite distingué comme l'un des meilleurs ministres des finances qu'ait eu le pays, et son sens de la discipline fiscale face aux fréquentes pressions des milieux de l'ANC lui ont acquis le respect des acteurs financiers. L'un et l'autre, mutuellement dépendants, portent ensemble la responsabilité de veiller à ce que le nouveau système d'objectifs concernant l'inflation ne devienne pas une gêne pour les marchés. Ils sont aujourd'hui de grands commis de l'Etats, personnages indépendants, qui se sont rapidement révélés compétents et adeptes d'une orthodoxie plutôt conservatrice en matières fiscale et monétaire.

On doit citer également le rôle joué par le Cosatu (Congress of South African Trade Unions), la centrale syndicale qui est l'une des forces non-politiques les plus importantes du pays. Elle s'est imposée comme une force d'opposition politique tant que l'ANC était banni. Ses dirigeants les plus talentueux sont devenus ministres du nouveau pouvoir, tout comme ceux de l'ANC et du SACP (South African Communist Party), et ils y défendent aujourd'hui le système économique «conservateur» auquel ce nouveau pouvoir s'est adapté.

Malgré les désaccords fréquents entre ces trois organisations dans les années passées, il semble bien que les sujets qui maintiennent leur alliance politique lors des élections sont plus forts que leurs différences, et que celle-ci a des chances de se maintenir à l'avenir. Cette alliance agit comme un frein sur la volonté du gouvernement de réformer le marché du travail, d'accroître les privatisations et de maintenir l'ouverture de l'Afrique du Sud sur les forces économiques internationales. En revanche, elle a contribué à rendre le climat social plus stable et a permis d'aboutir à certains accords sectoriels favorables à une meilleure compétitivité globale.

D'autres institutions économiques clés méritent d'être citées: 
- $\quad$ Le Conseil Economique National du Développement et du Travail (National Economic, Development and Labour Council NEDLAC): toutes les mesures législatives concernant l'économie et le travail doivent être légalement présentées pour avis aux quatre chambres (travail, finances publiques, commerce et industrie) de cette organisation qui se réunit régulièrement et doit chercher à aboutir à un certain consensus. Y sont représentés: le gouvernement, les organisations syndicales de travailleurs, les organisations professionnelles d'employeurs, et (dans la chambre du développement) les représentants des «communautés». L'Afrique du Sud post-apartheid a déjà une longue histoire de consultation sur les politiques du pays. Le gouvernement semblant trouver aujourd'hui que cette obligation de participer au NEDLAC est un fardeau, l'avenir de ce Conseil est incertain; les chambres du travail, du commerce et de l'industrie continuent pourtant de jouer un rôle majeur dans la formulation des politiques conduites par le gouvernement.

Le Conseil du Millenium: c'est un forum paritaire de discussion et de négociation des grands choix politiques, composé de représentants expérimentés des employeurs et des travailleurs.

- La Business South Africa (le monde des affaires sud-africain) est une organisation patronale qui exprime les intérêts des principales organisations d'employeurs sur les sujets politiques du moment. Son principal rôle est de participer au NEDLAC.

La Chambre des Affaires Sud-Africaines (South African Chamber of Business SACOB) et l'Institut du Commerce Africain (Afrikaanse Handelsinstituut AHI) sont des fédérations qui traitent de questions commerciales et de politique; elles sont historiquement dominées par le monde des affaires blanc.

- La Chambre de Commerce Nationale Fédérée (National Federated Chamber of Commerce $N A F C O C$ ) est la fédération principale des hommes d'affaires noirs. Une fusion entre NAFCOC et SACOB est imminente, mais des tensions subsistent entre les deux organisations.

\section{Les Equipements Publics et Privés d'Infrastructure}

L'Afrique du Sud a hérité de son passé de «puissance industrielle blanche» une masse considérable d'infrastructures modernes et régulièrement entretenues, dans le domaine des équipements urbains, des transports et des communications, qui sont certainement les meilleurs ou parmi les meilleurs de toute l'Afrique. Seule la Poste, service public depuis 1991, a connu des difficultés financières et techniques considérables, ce qui a provoqué l'arrivée sur le marché d'un bon nombre d'opérateurs privés (tels que Post Net) et les opérateurs de courrier international habituels. ${ }^{17}$

17 La compagnie néo-zélandaise Transend a été appointée en 1999 pour aider la société nationale à se gérer, mais le contrat a été rompu en 2002 sur la base de soupçons de corruption. 


\section{Les équipements urbains}

n'ont rien à envier à leurs homologues des pays du Nord (Europe, Amérique): bâtiments des services publics (et pas seulement dans les deux capitales, Pretoria et Le Cap); réseaux de voies routières et périphériques, gares ferroviaires, aéroports; réseaux de distribution d'eau, d'électricité, et d'assainissement, etc.

Seules les banlieues des grandes villes, de plus en plus étendues, souffrent chroniquement d'une absence ou d'une insuffisance de ces équipements.

\section{Les moyens de transports}

Les infrastructures de transport, principalement pour les activités économiques, sont très bien développées et certainement les meilleures d'Afrique: ports vastes et bien équipés, réseau routier, lignes aériennes (notamment avec l'Europe et les USA, mais de façon croissante avec l'Asie et le reste de l'Afrique). Cependant, du fait de la discrimination raciale, ces réseaux de transport ont été presque exclusivement conçus pour relier entre elles les villes et les zones d'habitation blanches. Le résultat est que le réseau de routes secondaires rurales est très pauvre, de même que les liaisons par route ou par rail entre les banlieues noires des villes (townships) et entre les «patries ethniques» (homelands). Le taxi «informel» dans les zones urbaines a été un foyer de violences et de crimes, souvent motivés par des luttes intestines au sein de cette activité, que le gouvernement a du mal à réglementer.

\section{Les télécommunications}

L'Afrique du Sud est le plus vaste marché des télécommunications de l'Afrique, et connaît un bouleversement à la suite de l'expiration, (en mai 2002) de la période d'exclusivité (garantie en 1997) accordée à l'opérateur national sur lignes fixes TELKOM: l'arrivée d'une série de services vocaux ou écrits électroniques. ${ }^{18}$ Depuis cette semi-privatisation, beaucoup de choses ont changé: avec les $10 \mathrm{mn}$ d'abonnés, les opérateurs de téléphonie mobile desservent près de deux fois plus de clients que l'opérateur de lignes fixes, et ont provoqué une réduction absolue du réseau de ce dernier en $2001 .^{19}$

Le marché du traitement de données et des services Internet s'est également accru de manière considérable. Le gouvernement prévoit d'accorder une licence à un deuxième opérateur national

18 L'exclusivité avait été accordée à Telkom après qu'une part de 30\% de son capital de Telkom eut été vendue à un consortium comprenant un transporteur US (SBC) et un opérateur malaysien (Telekom Malaysia), en échange d'objectifs élevés de croissance et de modernisation. En dépit des bonnes intentions affichées pour justifier la période d'exclusivité - le monopole de Telkom devait lui permettre de restructurer et d'étendre les services du téléphone à des zones souvent non rentables - les compagnies privées l'ont considérée comme le principal obstacle à une croissance plus rapide du marché sud-africain de télécommunications.

19 L'arrivée d'un $3^{\text {ème }}$ opérateur cellulaire en 2001 devrait porter le nombre d'utilisateurs de téléphones mobiles à plus de $15 \mathrm{mn}$ dans les cinq prochaines années. 
(SNO) qui utiliserait les réseaux de Transtel (l'unité de télécommunication du principal groupe de transport du pays, Transnet) et Esi-Tel (l'unité de télécommunication du distributeur d'électricité Eskom).

La South African Broadcasting Corporation (SABC), chaîne nationale de télévision, est officiellement indépendante, mais certains signes tendent à prouver que l'ANC accroît son contrôle sur la société. Elle émet sur trois canaux qui fournissent des programmes corrects mais pas de très haut niveau. Il existe aussi un service codé très populaire, appelé M-Net, qui diffuse dans d'autres parties de l'Afrique. La télévision par satellite a été lancée avec succès, et le premier canal terrestre privé d'Afrique du Sud, e-TV, a commencé d'émettre en 1997: il fournit le service d'information télévisée le plus indépendant du pays.

\section{LES MESURES PRISES ET A PRENDRE}

Depuis l'installation du nouveau régime d'intégration raciale, en 1994, toute une série de mesures ont été prises qui visent en particulier à réduire la pauvreté et améliorer les conditions d'existence de la population jadis marginalisée du pays. Des réformes structurelles de grande envergure ont été réalisées ou entreprises; de vastes programmes d'action ont été élaborés puis lancés, certains devant s'étendre sur plusieurs années. Bon nombre de ces réformes sont en cours d'application ou sont encore en discussion, et il est bien trop tôt pour en dresser un premier bilan. Il n'est même pas question, dans le cadre de cette communication, de les passer toutes en revue.

Globalement on peut dire que l'African National Congress (ANC) a d'abord adopté diverses politiques économiques favorables aux investisseurs. En matière fiscale, le gouvernement a cherché à rendre la politique fiscale plus crédible et plus prévisible en esquissant des programmes de dépenses sur une série d'années, et constitué une équipe de conseillers pour la définition de la politique économique et du code des investissements, composée d'hommes d'affaires internationaux de très haut niveau.

Parmi les changements les plus importants introduits par le gouvernement ANC, il faut citer l'introduction d'une législation du travail progressiste, qui, entre autres choses, encourage les entreprises à se constituer une force de travail (c'est à dire des «ressources humaines») «racialement représentative» et à mettre en œuvre des programmes de développement des qualifications, ainsi que d'une législation qui prévoit le transfert de titres de propriétés aux «populations désavantagées par la discrimination raciale». Ces mesures ont toutefois été critiquées par le secteur privé parce qu'elles porteraient atteinte à la liberté d'entreprendre et au droit de propriété, et décourageraient les entrepreneurs d'investir.

On tentera seulement, ici de présenter les principaux programmes ou mesures prises, en les classant de la façon suivante:

- Les grands programmes macro-économiques

- Les mesures de redistribution des emplois et des richesses 
- Les mesures d'amélioration de la «gouvernance» du pays par la régionalisation

\section{A Les Grands Programmes Macro-Economiques}

\section{La réflexion préalable}

Dès avant l'élection de 1994, le gouvernement provisoire ANC avait constitué un Forum Economique National chargé d'obtenir un consensus sur la définition d'une politique économique, et composé de façon tripartite (le Parti National de l'époque de l'apartheid, des organisations patronales, et des organisations de travailleurs, dont les délégations comprenaient des membres de l'ANC et du SACP communiste); il a été plus tard institutionnalisé sous le nom de NEDLAC. Il comprenait donc des personnalités venant d'horizons complètement opposés: des penseurs de la gauche radicale, des représentants de la droite conservatrice, des bureaucrates de l'époque de l'apartheid, et de nouveaux politiciens (certains tout juste sortis de prison). Les débats sur les modèles macro et microéconomiques ont été larges, ouverts et pragmatiques, et des expertises ont été demandées un peu partout dans le monde: FMI, Banque Mondiale, Organisation Internationale du Travail... Les opinions des principaux acteurs de cette discussion/confrontation se sont cristallisés au cours de cette période: l'ANC s'est défait de sa conception dirigiste antérieure, particulièrement son soutien aux nationalisations, et a accepté la nécessité d'affronter la compétition dans une économie mondiale grâce des politiques favorables aux investisseurs. L'essentiel de la politique de libéralisation a, de fait, été mise en œuvre avant même que l'ANC accède au pouvoir.

\section{Les documents clés: le RDP et le GEAR}

a) Le Programme de Reconstruction et de Développement (The Reconstruction and Development Programme RDP), lancé en 1994, a été la première clé de voûte de la politique économique du gouvernement, destinée à redresser certaines des plus criantes inégalités de l'économie et relancer les perspectives de croissance après 1994. Progressif dans sa conception, il projetait une dépense de l'Etat de 37,5 md de rand (10,6 mds de \$ US au taux de change de l'époque) sur une période de 5 ans, sur des programmes d' «élévation» sociale. Toutefois, ce document de politique se préoccupait peu de savoir si la croissance ainsi obtenue serait durable et soutenue. En outre, le ministère spécialement affecté à ce programme manquait d'autorité politique et «opérationnelle» pour coordonner les départements ministériels concernés, et a été finalement supprimé. La responsabilité de la réalisation du RDP a été transférée au cabinet du vice-président Thabo MBEKI. En pratique, le RDP a finalement été intégré dans les allocations budgétaires normales et les fonctions des départements sociaux, mais la vision qu'il représentait faisait toujours partie intégrante de la politique économique du gouvernement. Le COSATU essaye encore fréquemment de le ressortir des cartons et lui restituer son rôle de document de base, pour remplacer l'actuelle stratégie macro-économique prédominante.

b) Le GEAR: Croissance, Emploi et Redistribution: une Stratégie Macro-Economique (Growth, Employment and Redistribution - a Macroeconomic Strategy): c'est le titre du document que lance le gouvernement, en juin 1996, pour lutter contre le déferlement d'un débat violent sur la façon de 
surmonter la crise du sous-emploi en Afrique du Sud et de porter son économie à un niveau plus élevé de croissance créatrice d'emploi. Il rejette l'idée d'instituer un salaire minimum national et une politique fiscale «expansionniste» pour atteindre ces objectifs, et considère qu'une dépréciation «réelle» du rand ${ }^{20}$ peut relancer la croissance si elle est parallèlement soutenue par une ferme politique monétaire anti-inflationniste. En plus, et au-delà des engagements pris en matière de privatisation et de réorientation de la dépense publique au profit des pauvres, les propositions-clés de cette politique sont énoncées: consolidation fiscale plus rapide, «flexibilité régulée» sur le marché du travail; réductions accélérées des droits de douane; dépréciation accélérée pour stimuler de nouveaux investissements dans l'industrie; exemption de taxes pour les nouveaux projets approuvés concernant des régions et des industries dont la liste est dressée; et franchissement d'une étape supplémentaire dans le processus de libéralisation progressive du contrôle des changes, comportant la suppression des restrictions imposées pour l'accès au crédit intérieur des investisseurs étrangers.

c) Une nouvelle stratégie «intégrée» pour l'industrie: Le gouvernement a défini sa stratégie globale pour l'industrie - the Integrated Manufacturing Strategy IMS - en avril 2002; elle est basée sur l'analyse de deux tendances concurrentes qui se sont manifestées dans le secteur manufacturier au cours des cinq dernières années: la croissance de l'industrie automobile, et le déclin de l'industrie textile (fibres, tissage et confection). Tirant les leçons de ces tendances, cette stratégie a pour objet de promouvoir le développement de huit branches clés de l'industrie: textiles et confection, transformations agro-alimentaires, minerais et métaux, tourisme, véhicules automobiles et transports, artisanat, chimie, bio-technologie et industries de haute technologie. Les industries choisies reflètent bien les différentes facettes de l'économie sud-africaine, qui vont d'une forte base agricole et minière à des secteurs valorisant les nouvelles techniques de l'information/communication. Mais cette stratégie reconnaît aussi sa structure duale, avec des secteurs de technologie ancienne ou rudimentaire et «intensives en emploi - c'est-à-dire grosses consommatrices de main d'œuvre de technicité moyenne et de bon savoir-faire manuel: artisanat, textiles, tourisme... - et d'autres «intensifs en capital» et (ou) en innovations scientifiques de pointe (branches informatisées et automatisées, nouvelles technologies...) qui «consomment» une main d'œuvre hautement qualifiée. Cette stratégie ne prévoit pas que le gouvernement s'implique dans des projets particuliers de chaque secteur, mais définit des objectifs généraux pour le Ministère du Commerce et de l'Industrie, visant à promouvoir la compétitivité de ces industries et à leur fournir des soutiens en infrastructure et logistique.

\section{Les revenus et la fiscalité}

On peut affirmer que la politique économique de la période post-apartheid a été dominée par la question de la politique fiscale. Le Ministre des finances, Trevor Manuel, a préconisé une politique fiscale «conservatrice», consistant à réduire drastiquement le déficit budgétaire et à stabiliser la

20 C'est à dire à un taux plus fort que celui de l'inflation. 
dette du gouvernement pour établir une base durable pour la dépense publique. Les rentrées fiscales ont régulièrement dépassé les objectifs, grâce à la restructuration réussie des services fiscaux sudafricains et à une assiette fiscale notablement élargie. Les dépenses ont été gérées dans une perspective de moyen terme, grâce à un budget étalé sur trois ans destiné à faciliter la programmation de leurs dépenses par les différents ministères et à instaurer plus de stabilité dans leur processus de dépense

Après une période impressionnante de sept années de consolidation des finances publiques et de contraintes fiscales, la politique fiscale du gouvernement se situe, depuis l'exercice 2001/02 dans une plage modérément expansionniste. Cela autorise $\mathrm{M}$ Trevor Manuel à réduire les impôts et à accroître la dépense dans deux budgets consécutifs (2001/02 et 2002/03) - un résultat sans precedent. $^{21}$

\section{Les attentes insatisfaites}

a) Un consensus difficile: Le gouvernement s'est très largement employé à obtenir et conserver le soutien de l'ANC à sa philosophie économique: chaque année il indique avec insistance dans son budget que la stabilisation de la conjoncture est une condition préalable indispensable à une politique sociale généreuse. Sa conception des choses a le soutien des organisations professionnelles, qui estiment cependant trop lent le processus de privatisation et trop onéreuse la législation en faveur des travailleurs. Pourtant, les deux partenaires de l'ANC au sein de l'alliance (SACP et COSATU) ont vigoureusement critiqué cette conception générale, jugée conservatrice. Ils maintiennent l'idée qu'elle sous-estime le rôle de la demande pour stimuler le redressement, et que la poursuite d'une politique monétaire et fiscale rigoureuse condamnera le pays à un niveau bas de productivité. Cette différence sous-jacente de conception économique provoque des tensions au sein de l'alliance, qui périodiquement s'élèvent et s'enflamment à l'occasion de débat sur le budget, les salaires du secteur public ou la privatisation. Cependant le gouvernement n'a pas dévié de la direction économique générale décrite dans le GEAR. ${ }^{22}$

b) Le risque d'un retour en arrière: le principal succès du GEAR a été de stabiliser l'économie. Toutefois, il n'a pas atteint ses objectifs essentiels: avant toute chose, mettre l'économie sudafricaine sur une trajectoire de croissance plus haute (disons de plus de 6\%) et créatrice de 400000 emplois nouveaux avant 2001. En réalité, la croissance a été lente et le chômage est resté

21 Les Perspectives de Dépenses à Moyen Terme (Treasury's Medium-Term Expenditure MTEF), publiées par le Trésor fin octobre 2002, prévoient une croissance des dépenses de 4,7\% pour les trois prochaines années; la décision du gouvernement d'accepter un déficit budgétaire modérément expansionniste de 2,1\% du PIB en 2002/03 a été bien accueillie par le monde des affaires. Les dépenses publiques s'accroîtront de 11,1\% et les recettes de 10,1\%. Les dépenses sociales augmenteront de 11,8\%, avec des taux de hausse encore plus élevées pour les dépenses de protection sociale (+20\%), celles de logement et de services des communautés (+18\%). On peut regretter que les dépenses d'éducation et de santé ne croissent que de 8\%.

22 De façon anecdotique et probablement involontaire, ce sigle désigne en anglais la «vitesse» - celle qu'il faut judicieusement choisir dans une boîte à vitesse pour que le moteur tourne rond. 
obstinément élevé, ce qui a fourni des munitions aux partenaires de l'ANC dans l'alliance (COSATU et SACP), qui ont condamné l'orthodoxie du GEAR au vu de ses échecs. Un mécontentement populaire au sujet de la politique sociale augmenterait le risque d'un renversement de la politique économique.

La volonté politique de conserver les orientations économiques actuelles reste cependant forte. L'opinion se fait jour qu'une partie des raisons qui n'ont pas permis au GEAR d'atteindre ses objectifs étaient hors du contrôle du gouvernement - la crise des marchés émergents en 1997, le délai nécessaire entre la mise en œuvre de politiques nouvelles et la perception de leurs benefices. ${ }^{23}$ Cependant, les analystes prétendent aussi que les éléments clés de la stratégie du GEAR n'ont pas été convenablement mis en place, en particulier la rationalisation d'un secteur public hypertrophié (dont les employés absorbent l'essentiel du budget des dépenses sociales), la réforme du marché du travail (qui reste inadéquate) et le rythme des privatisations (qui reste trop lent).

c) Une stratégie à repenser, de nouveaux moyens d'action à trouver. Le GEAR a été conçu comme une stratégie de moyen terme, et son opération s'achevait en 2000. Depuis, il n'a pas été mis à jour ou remplacé par une nouvelle stratégie, ce qui laisse l'économie du pays sans programme d'action globale.

L'engagement du gouvernement dans une discipline fiscale et monétaire est à l'évidence une condition nécessaire mais pas suffisante pour relancer la croissance. Les micro-stimulants mis en place dans le budget 2001 et 2002 ne combleront pas cette insuffisance d'action globale. Un risque de retournement de la politique économique sous la pression populaire ne pourra être évité que si, parallèlement à une stratégie de croissance redéfinie, le gouvernement fait davantage dans le domaine social, en sorte de dissiper l'impression que rien ne change dans ce domaine. La tâche la plus difficile sera d'améliorer la capacité et l'efficacité des institutions chargées de la protection, de la solidarité et de l'entraide sociales.

\section{B Les Mesures de Redistribution des Emplois et des Richesses}

Le gouvernement s'est efforcé d'intervenir, de façon assez radicale parfois, dans trois directions:

(1) La réforme des règles régissant l'emploi et le fonctionnement du marché du travail;

(2) Un certaine redistribution des titres de propriété en faveur des «populations défavorisées par la discrimination raciale»;

(3) Les privatisations.

23 Le phénomène bien connu traduit pas la courbe en forme de $\mathrm{J}$. 


\section{Les lois sur le fonctionnement du marché du travail}

Le FMI, dans sa consultation annuelle sur l'état de l'Afrique du Sud de mai 2001, a insisté sur le fait que la réforme du marché du travail (contrats d'embauche, durée du travail, congés payés, licenciement, conventions collectives, droit de grève et négociations salariales...) était essentielle pour remédier au taux très élevé de chômage. Des modifications législatives très attendues, et approuvées par les employeurs et les travailleurs salariés, ont été adoptées en 2002. Ces amendements devraient globalement avoir un effet neutre sur le marché du travail - à la différence du projet originel du gouvernement, qui se rangeait au point de vue des chefs d'entreprise selon lequel le marché du travail sud-africain était trop rigide, une des causes principales de la faible croissance de l'économie du pays. Pourtant, les milieux d'affaires ont accordé leur soutien à la nouvelle version des amendements, probablement pour la raison que le principal sujet de litige dans cette législation - l'extension automatique à toutes les branches des accords négociés au niveau central - avait été retiré, et que, pour d'autres aspects, il a été finalement estimé qu'ils ne justifiaient pas de mettre en péril les relations industrielles existantes. Toutefois, les nouvelles dispositions amélioreront l'efficacité du marché du travail selon la façon dont la Commission de Conciliation, Médiation et Arbitrage fonctionnera pour résoudre les conflits. Sur un point plus litigieux, les syndicats ont obtenu satisfaction: les chefs d'entreprise devront les consulter sur les licenciements envisagés, et les syndicats auront désormais le droit de déclencher des grèves contre les licenciements.

\section{Les mesures en faveur de l' «enrichissement économique» des Noirs}

a) L' «enrichissement économique des Noirs», ${ }^{24}$ c'est à dire le transfert de propriété des industries clés d'Afrique du Sud aux populations désavantagées par la discrimination raciale (apartheid), a été mis au premier rang des préoccupations publiques en 2002. Le gouvernement sudafricain avait porté peu d'attention aux recommandations de la Commission du «BEE» au début de l'année 2002, jusqu'au moment où de nouvelles propositions - radicales - pour l'industrie minière faites par cette Commission sont apparues au grand jour à l'occasion d'une fuite d'information concernant le projet gouvernemental de charte traitant du transfert de propriété dans cette industrie. Depuis lors la charte a été révisée après consultation avec ses représentants, mais la position initialement prise par le gouvernement montrait son intention de mettre en pratique les principes jusque là assez vagues de renforcement du pouvoir économique ou «enrichissement» de la population noire. Le gouvernement poursuit toujours sa réflexion sur la question générale du «BEE», mais, s'efforçant de prendre ses distances à l'égard des méthodes du Zimbabwe, il insiste sur fait que cette politique sera menée en Afrique du Sud de manière réfléchie, légale et transparente, et que tout transfert d'avoirs sera pleinement négocié. Au cours du congrès national de l'ANC en

24 Traduction libre pour «the Black Economic Empowerment (BEE)»; il serait plus précis de dire «l'accession des noirs à la propriété et au pouvoir». 
décembre 2002, ce parti a insisté sur l'importance d'une application réfléchie de cette politique de BEE, dans l'intérêt de la stabilité politique générale du pays.

b) S'agissant de l'industrie minière, la version finale de la loi sur le Développement des Mines et des Ressources Pétrolières a été votée par le parlement en juin 2002, mettant un terme à un long processus législatif, et transférant tous les droits d'exploitation minière à l'Etat. Ce dernier accordera des licences pour la prospection et l'exploitation des mines - à l'image des législation européennes, et à la différence des lois nord-américaines sur la propriété des mines. L'objectif en filigrane est d'ouvrir le secteur à la concurrence étrangère et d'encourager le développement de groupes miniers possédés par des Noirs. Les aspects controversés de la nouvelle loi concernent la sécurité ou durée garantie des titres de concession (ou tenures) qui seront accordés, et les pouvoirs discrétionnaires du ministre des minerais et de l'énergie en matière d'octroi et de retrait des licences de prospection et d'exploitation. Les compagnies minières, qui rappellent qu'elles sont des opérateurs qui agissent sur le long terme, et qui estiment que la garantie de durée de leurs titres de concession n'est pas assurée dans la nouvelle loi, ont averti le gouvernement que «des millions de dollars (?) d'investissement allaient être compromis».

Maintenant que la loi est passée, les compagnies minières peuvent recourir aux tribunaux pour la défense de leurs droits, et le gouvernement risque de faire face à une sanction constitutionnelle si la question d'une compensation pour les droits miniers «perdus» n'est pas clarifiée.

Les compagnies minières avaient à peine digéré le Mineral \& Petroleum Resources Bill qu'une autre fuite d'information se produisait au sujet de la charte du ministère des minerais et de l'énergie, qui proposerait que $51 \%$ des titres de propriété miniers soient transférés aux mains des «SudAfricains historiquement désavantagés» dans la décennie à venir. Cette information a provoqué la panique sur les marchés financiers, des ventes de titres (en particulier des parts d'actifs miniers) et une forte sortie de capitaux flottants en juillet et août 2002, provoquant une dégringolade des cours. Une version finale de la charte a été publiée en octobre 2002, obtenue après consultation des compagnies et des travailleurs, qui présentait le consensus obtenu à la fois sur l'étendue et la vitesse de la réforme. Cette charte propose que la propriété de 15\% des richesses minières du pays soient transférée à des Noirs d'ici cinq ans, et 26\% d'ici dix ans. L'industrie minière a accepté de consacrer 100 mds de rands à la réalisation de ce transfert.

c) C'est peut-être dans l'agriculture que l'héritage de la discrimination raciale est le plus visible. Le gouvernement de l'après-apartheid a hérité d'une structure de la propriété des terres très inégalitaire, créée par une législation du début de $20^{\mathrm{e}}$ siècle, et renforcée par les gouvernements de la période de l'apartheid, qui ont tenté d'entasser $75 \%$ de la population sur 13\% des terres. La réforme foncière est donc une question essentielle, devenue encore plus cruciale depuis les occupations de terres commanditées par le gouvernement qui se sont produites au Zimbabwe.

Le gouvernement sud-africain a fait très attention de conduire son programme de réforme foncière dans le strict respect des lois. En 1994, l'ANC s'était donné pour objectif d'attribuer 30\% 
des terres agricoles à des fermiers noirs d'ici 1999, en leur transférant des terres possédées par l'Etat, en reprenant possession de fermes détenues par des fermiers blancs gravement endettés, et en achetant des fermes à l'issue de longs règlements judiciaires. Mais ces objectifs n'ont pas été atteints, les retards administratifs et les luttes politiques intestines entre les bureaucrates ayant entravé le processus.

Le programme de restitution des terres du gouvernement, dont l'objectif est de traiter les 69000 demandes qui avaient été déposées avant la date butoir en 1998, a récemment regagné du terrain: seules 12 demandes avaient été traitées en 1998; à la mi-2001 ce chiffre avait atteint 12 000. Mais un rapport de l'Université de Western Cape a objecté que la plupart des cas traités ont été réglés en argent et non pas en terre, et estimé qu'il faudrait encore 150 ans pour régler les cas restants. En 2001, le ministre de l'agriculture (Thoko DIDIZA), après consultation de la Banque Mondiale, a remplacé l'objectif de redistribution de terres rapidement récupérées par celui consistant à développer une classe de noirs fermiers-marchands (c'est à dire vendeurs de leurs produits) à plein temps. La controverse porte sur le point de savoir comment le gouvernement va manœuvrer avec le système foncier communal des anciennes «patries ethniques» (homelands), où l'administration reste entre les mains à la fois des autorités locales et tribales. Plus fondamentalement, il est reproché à cette politique de ne pas prendre à bras le corps le problème critique des familles rurales pauvres sans terre.

\section{Les privatisations}

Quelques observations doivent être faites au sujet des privatisations, qui - dans la mesure où elles reviennent, par ventes de titres sur le marché financier, à confier à des particuliers une partie au moins de la propriété du capital de sociétés jusque là nationales - semblent aller à rebours de la réforme précédemment évoquée pour les activités minières, si rien n'est prévu pour réserver une partie de ces titres aux «populations historiquement déshéritées».

Le gouvernement, à la fin du siècle dernier, a admis la nécessité de privatiser, à la fois pour améliorer la fourniture de certains services et pour procurer des recettes à l'Etat. Le rythme (lent) des privatisations adopté par le gouvernement - motivé par la résistance politique à laquelle elles se heurtaient - a affecté la confiance des investisseurs. En vérité le gouvernement avait de bonnes raisons d'agir avec prudence. La définition du statut légal des compagnies relevait des systèmes opaques de la période de l'apartheid. De plus, des restructurations importantes étaient requises pour remédier à l'inefficacité de certaines compagnies, certaines d'entre elles étant engluées dans de lourds endettements. Cependant, le temps de cette restructuration est maintenant passé, et la pression pour réaliser les privatisations s'est accrue, venant non seulement des investisseurs mais également du fisc qui souhaiterait profiter du produit des ventes d'actions.

Le gouvernement a publié en août 2000 un document très attendu intitulé «un calendrier accéléré pour la restructuration des entreprises d'Etat» (An Accelerated Agenda Towards the Restructuring of State Owned Enterprises); mais il reste très imprécis sur les modalités et très large 
dans les projections en matière de calendrier, ce qui accroît les incertitudes sur le marché. Le COSATU et d'autres centrales syndicales ont mené en octobre 2002 leur seconde grève nationale contre le programme des privatisations en octobre 2002. La question reste posée de savoir si les procédures au sein de l'alliance (des partis politiques au pouvoir) sont capables de régler ce désaccord avec les syndicats.

Les 18 mds de rand de recettes de privatisation programmées par le gouvernement pour 2001/2002 ont été reportées en 2002/2003, mais il est encore peu sûr que ce volume soit atteint. De 1997 à 2001, les privatisations réalisées ont produit 12,4 mds de rands (provenant principalement d'acquéreurs étrangers) qui ont été utilisés à réduire la dette du gouvernement - un choix très justifiable, mais politiquement difficile à faire accepter au regard d'une revendication en faveur d'un accroissement de la consommation. Mais le gouvernement a contourné l'objection (et continuera sans doute à le faire) en insistant sur le fait que la privatisation est une part centrale de sa stratégie en faveur de l'enrichissement des Noirs, l'Etat se procurant à la longue, par les privatisations, des ressources nécessaires à l'indemnisation des propriétaires du capital des groupes miniers - et peutêtre un jour d'autres activités (industrielles ou de services) initialement nationalisées? - dont une partie des titres seraient cédés (gratuitement ou à bas prix) à de nouveaux propriétaires issus des populations «historiquement défavorisées».

\section{A Gouvernance et Régionalisation}

Le dernier domaine où des mesures sont susceptibles de contribuer à réduire la mise à l'écart (ou marginalisation) des populations historiquement défavorisées est peut-être celui d'une gouvernance du pays cherchant à se rapprocher de ces populations, en donnant plus de pouvoirs aux autorités locales directement en contact avec elles, et en favorisant le développement diversifié des provinces et districts.

\section{La réorganisation des provinces et des conseils locaux}

a) les provinces: Comme beaucoup d'autres institutions d'Afrique du Sud, le gouvernement des régions a été réorganisé après 1994. Neuf gouvernements provinciaux nouveaux ont été créés, dans des régions au découpage nouveau - elles ont notamment absorbé les anciennes «patries ethniques» (anciens «bantoustans» ou «homelands» auxquels le gouvernement blanc avait octroyé une indépendance formelle) - qui, pour certaines, ont changé de nom. Ce sont aujourd'hui, du Nord au Sud, le Limpopo, le Gauteng (ancien Witwatersrand), le Mpumalanga, le North West, le Free State, le KwaZul-Natal, le Northern Cape, l'Eastern Cape et le Western Cape.

Pour la première fois dans leur histoire, ces provinces se sont vu attribuer non seulement des pouvoirs administratifs, mais aussi certains pouvoirs législatifs limités. Chaque région envoie dix représentants au Conseil National des Provinces, assemblée parlementaire. En 1999, l'ANC était encore largement prédominant dans la plus part des provinces, deux d'entre elles seulement étant gouvernées l'une par l'Inkhata Freedom Party (le KwaZulu-Natal) et l'ANC dans une difficile coalition, l'autre (le Western Cape) par l'Alliance démocratique (DA) jusqu'en fin 2001, date à 
laquelle le NNP (New National Party) a quitté cette alliance et choisi de partager le pouvoir avec l'ANC.

La gestion des finances de ces provinces s'est remarquablement améliorée: elles ont enregistré un net déclin de leur endettement envers le secteur privé depuis 2001. Le principal obstacle à une meilleure fourniture de services sociaux tient aux contraintes budgétaires que subit le niveau provincial du gouvernement, dont les allocations en capital se sont cependant notablement améliorées en 2001/2002.

Au sein du gouvernement et de l'ANC au niveau national, des préoccupations s'expriment concernant le coût et l'efficacité des provinces et la diffusion du pouvoir politique. Ce sentiment se manifeste par la réticence du gouvernement central à attribuer aux provinces des pouvoirs leur permettant d'accroître leur propres recettes. Un organisme permanent, la Commission Financière et Fiscale, est chargé de conseiller le gouvernement central sur la meilleure façon de répartir entre les provinces les recettes collectées au niveau central, mais en pratique elle a peu d'influence. En réalité, les provinces ont créé leurs propres organes politiques qu'il sera difficile de déloger.

b) les conseils locaux: Le gouvernement local représente une sphère de décision et de gestion autonome du gouvernement central. Aux premières élections locales multi-raciales de 1995/96, des groupes minoritaires dans chaque district local se sont vu reconnaître une représentation. Les Conseils locaux ont ensuite dû faire face au problème de la fusion des districts racialement séparés au sein d'autorités locales unifiées, et de l'abandon de la culture du refus de payement des loyers et services, qui était devenu avant 1994 une forme de protestation politique dans les districts «africains».

En novembre 2000, la représentation garantie des minorités a été supprimée, le nombre de conseils locaux a été considérablement réduit, et des conseils pour grandes cités ont été créés au Cap, à Durban, Johannesburg, Pretoria, et quatre autres métropoles. Cette rationalisation avait pour objet de réaliser des économies d'échelle et d'améliorer le rapport coût/efficacité des services locaux. Les nouvelles structures sont considérablement plus puissantes, gèrent d'importants budget, et deviennent les structures clés pour la fournitures de services, au point qu'on s'attend à ce qu'elles éclipsent les gouvernements provinciaux, ce qui risque d'entraîner un débat sur leur avenir.

Les principaux défis auxquels doivent faire face ces nouvelles structures locales de pouvoir concernent l'abolition du refus de payement des services dans les zones «noires» et le renforcement de leur capacité à fournir des services de qualité. Le comportement des agents économiques privés sera déterminant dans cette affaire, en dépit de l'opposition des syndicats de fonctionnaires locaux. Un élément nouveau important a été, en 2002, l'étude recommandant que soit créé un service public unique et que des fonctionnaires nationaux et provinciaux soient affectés au gouvernement local.

\section{Le programme des zones et initiatives de développement régional}

Dans son souci de mieux répartir et réguler le développement industriel dans le pays, le gouvernement central a encouragé les opérateurs privés à s'impliquer dans de nouveaux projets 
d'infrastructures, dénommés Initiatives de Développement Régional (Spatial Development Initiatives SDI), dans des zones de développement industriel potentiel (Industrial Devlopment Zones IDZ), où l'accent doit être mis tout particulièrement sur les actions d'enrichissement des populations historiquement déshéritées (black empowerment).

Le programme des SDI a pour objectif de développer le potentiel économique de régions spécifiques d'Afrique du Sud, en utilisant les ressources publiques pour inciter le secteur privé à investir. Le programme consiste en huit SDI et douze zones IDZ. Ces dernières sont proches d'un aéroport ou d'un port international; ${ }^{25}$ elles sont destinées à offrir aux investisseurs un port d'accès facile, des infrastructures modernes, y compris des entrepôts pour importations et exportations, et des coûts faibles pour l'implantation de nouvelles enterprises. ${ }^{26}$

\section{CONCLUSION}

Il est naturellement trop tôt pour dresser un bilan général des actions entreprises qui ont pour but ou peuvent avoir pour effet d'améliorer régulièrement et durablement le sort des populations historiquement déshéritées (santé, éducation, formation et emploi, accès à la propriété et aux responsabilités), en sorte de réduire l'écart de revenu et de niveau de vie immense qui subsiste encore entre ces populations et celles qui ont hérité des richesses et du savoir accumulés pendant les décennies de discriminations raciale.

Nous avons cependant pu marquer, au passage, les progrès accomplis, et aussi les erreurs et les insuffisances observées dans leurs effets, comparés aux espoirs qu'on avait mis dans les politiques ou mesures adoptées. Ce qui nous semble transparaître dans ce tour d'horizon, c'est que la volonté politique de relever ce que l'on peut appeler le Grand Défi n'a, elle, pas fait défaut, et continue d'exister. Il est remarquable de constater qu'à chaque étape et devant les principales difficultés rencontrées, un consensus a toujours fini par s'imposer, tant l'objectif final semble partager par les principales forces politiques et syndicales. Avec les richesses naturelles et humaines évoquées en $2^{\mathrm{e}}$ partie, il nous semble que c'est surtout là que réside le principal atout de ce pays, et ce sont bien ces atouts qui nous permettent de rester résolument optimiste quant à ses chances de relever le défi à l'avenir.

25 Coega a déjà reçu son permis d'opérer, East London et Richards Bay doivent suivre.

26 Les principales SDI concernent les régions suivantes: le Corridor de Maputo (reliera le Gauteng, le Mpumalanga et le Mozambique: industries, tourisme, transport, dont une autoroute de Witbank à Maputo); Lubombo (routes et équipements touristiques entre Maputo et le KwaZulu-Natal); West Coast (le long de la côte Ouest du Cap: mines, tourisme et agro-alimentaire); Platinum (reliera le corridor de Maputo du Gauteng à l'autoroute du Kalahari au Botswana); Phalaborwa (complètera le corridor de Maputo en reliant Nelspruit au Mpumalanga et Phalaborwa dans la Northern Province); Richards Bay (au Nord du KwaZuluNatal, complètera la SDI de Lubombo, avec un terminal passager à Richards Bay). 